\title{
Les pays du Sud face au défi d'un espace financier euroméditerranéen
}

\author{
Jean-Pierre ALLEGRET ${ }^{*}$ et Bernard COURBIS ${ }^{* *}$
}

\section{Résumé}

L'objet du texte est de voir en quoi le choc du passage à l'Union Economique et Monétaire pourrait créer un relais entre les systèmes nationaux du sud de la Méditerranée et le processus de mondialisation financière. Dans la première partie, la dimension structurelle de l'espace financier euro-méditerranéen est identifié. Nous montrons que les conditions d'insertion internationale des pays du Maghreb ne leur permettent ni une stratégie d'intégration régionale, ni une stratégie de mondialisation. De l'articulation des balances des paiements entre l'Europe et le Sud de la Méditerranée est ensuite déduite la possibilité de faire émerger un système financier euro-méditerranén fondé sur le transfert des excédents courants du Nord au Sud. Dans la seconde partie, est approfondie la question de la formation d'un cet espace euro-méditerranéen en considérant son impact sur les systèmes financiers du Sud. Nous rappelons en quoi la création de l'euro va induire la formation d'un espace financier européen en insistant sur le fait que, compte tenu de la structure des paiements courants de l'Union européenne, l'euro sera une monnaie d'endettement. Après avoir souligné les conditions de l'ancrage à l'euro des monnaies du Maroc et de la Tunisie, nous analysons les principales mutations des systèmes financiers - ainsi que leurs limites - dans les deux pays.

\section{Abstract}

Mediterranean Economies coping the Challenge of an Euromediterranean Financial System.

The creation of the EMU will induce important consequences in the region. The purpose of this paper is to show how EMU could create a breach between Mediterranean financial systems and the financial globalisation process. In the first part, we identifiy the structural dimension of the euromediterranean financial system. The international insertion of the Maghrebian economies does not permit neither a regional strategy nor the worldwide strategy. Despite these difficulties, the articulation of balance of payments between Euro and South mediterranean countries can lead to the emergence of an Euromediterranean financial system. The integration of Mediterranean economies to EU could be the condition of the insertion of the first area in the global economy. This Euromediterranean financial system is based on financial transfers - owing to the European current surplus - between the two areas. In the second part, we study the impact of the formation of an Euromediterranean financial space on the Maghrebian financial systems. The main characteristic of this financial space is the fact that the euro will be debt money". This is a consequence of the surplus position of EU. The Maghrebian economies'anchor strategy to the Euro is examined as the progress and limits of the financial systems of Marocco and Tunisia in relations with the minimal conditions to fulfill to benefit of the European financial integration

\section{Introduction}

Les relations économiques entre l'Union européenne (UE) et les pays du sud de la Méditerranée ont pris une nouvelle tournure au début des années 90 en raison de la 
proposition européenne d'établir une zone de libre échange avec des pays du Maghreb et du Machrek $^{\mathrm{i}}$ et de la décision du Conseil européen de 1994 de renforcer la politique méditerranéenne. Deux éléments vont incarner cette nouvelle stratégie. D’une part, des accords d'association sont signés entre l'UE et les pays méditerranéens. Si l'objectif ultime est bien d'obtenir la création d'une zone de libre échange euro-méditerranéenne, le faible degré d'intégration économique régionale des partenaires de l'Union impose la tenue de négociations sur une base à la fois bilatérale et multilatérale nord-sud, mais non sur une base inter-régionale. Dès lors, les accords d'association s'interprètent comme la réalisation de zones de libre-échange bilatérales pour les produits industriels censées appuyer la formation d'une zone de libre échange globale ${ }^{\mathrm{ii}}$. D'autre part, la Conférence européenne de Barcelone, en novembre 1995, a confirmé ces objectifs en orientant les relations économiques entre l'Europe et la Méditerranée vers une libéralisation progressive des échanges de produits manufacturés et de services dans le cadre de l'Organisation Mondiale du Commerce, à l'horizon de 2010. Dans cette perspective, l'Union s'engage à soutenir les efforts de réduction des barrières douanières en accroissant parallèlement le financement communautaire en faveur des pays méditerranéens, participant ainsi au processus de mondialisation de ces économies.

Ainsi l'opposition multilatéralisme - régionalisme, bien présente, n'est pas nécessairement insurmontable. L'intégration régionale n'est pas incompatible avec une plus grande participation à l'échange mondial, dans la mesure où , comme le souligne Lawrence (1997), les nouveaux accords régionaux vont au delà de la libéralisation du commerce, pour concerner les services et les mouvements de capitaux. Ainsi, même si les accords passés entre l'UE et les pays méditerranéens semblent traditionnels, la réalisation de la monnaie unique met au premier plan la dimension financière des relations euro-méditerranéennes. Ce tryptique commerce-monnaie-finance va nous permettre d'examiner si le choc du passage à l'Union Economique et Monétaire pourrait créer un " relais " ou un " sas " entre des systèmes nationaux et la mondialisation financière. Plus précisément, il s'agit de voir en quoi la formation d'une zone monétairement unifiée modifie, par l'intermédiaire du mouvement d'intégration financière internationale, les perspectives ouvertes par les accords de libre-échange commerciaux. Les économies du sud pourraient ainsi entrer à la fois dans un processus de mondialisation de leurs relations commerciales et financières, dans le cadre de l'OMC, et dans un processus d'intégration régionale commerciale, financière et monétaire, dans le cadre de leur positionnement vis-à-vis de l'UEM.

Dans cette perspective, la notion d'espace financier euroméditerranéen est d'abord mise en évidence à partir d'une étude structurelle des échanges courants des pays du sud et de l'articulation de leur balance des paiements avec l'Europe. Ensuite, est envisagée l'évolution future de cet espace à travers l'analyse de l'impact de l'euro sur la poursuite de la mutation des systèmes financiers sud-méditerranéens. 


\section{La dimension structurelle de l'espace financier euro-méditerranéen}

L'observation des échanges des pays sud-méditerranéens, et du Maghreb plus particulièrement, comparativement à d'autres régions du monde conduit à un constat de blocage. En effet, il apparaît que ni la régionalisation, ni la mondialisation ne semblent réalisables pour ces pays (A). En revanche, l'articulation des balances des paiements entre les pays nord et sud-méditerranéens semble ouvrir la voie à une dynamique de développement propice à l'insertion internationale de ces derniers (B).

\section{A. Régionalisation et mondialisation : une double impossibilité \\ 1. L'absence d'intégration régionale}

L'insertion des pays sud-méditerranéens pourrait s'appuyer sur une stratégie de "régionalisme ouvert" selon l'expression de Bergsten (1997) dans laquelle l'intégration régionale sert de base à l'insertion des économies domestiques dans l'économie mondiale. Une telle stratégie s'apparente à celle suivie par les pays d'Amérique Latine depuis les années 80 avec la multiplication des accords régionaux. L'accord le plus important est le Marché commun du Sud - dit aussi MERCOSUR - impulsé en 1991 entre le Brésil, l'Argentine, l'Uruguay et le Paraguay, et deux pays associés (le Chili et la Bolivie). Les échanges intra-zones ont été multipliés par trois entre 1991 et 1995, illustrant par la même le dynamisme de l'intégration régionale.

Mais, comme le montre le tableau 1, une spécificité importante du Maghreb, par rapport à d'autres ensembles régionaux, est le très faible degré d'échanges intra-zones. Un tel constat interdit d'envisager une stratégie d'insertion dans l'économie mondiale fondée sur la régionalisation du commerce.

Tableau 1 Structure géographique du commerce extérieur des pays de l'Union du Maghreb Arabe. En \%.

\begin{tabular}{|lccccc|}
\hline & 1991 & 1992 & 1993 & 1994 & 1995 \\
\hline Importations & & & & & \\
Total UMA & 3,4 & 3,3 & 2,4 & 3,2 & 2,3 \\
Europe & 65,3 & 63,3 & 61,8 & 62,6 & 66,4 \\
Amérique & 12,6 & 11,4 & 15,3 & 14,2 & 12,7 \\
Asie & 15,9 & 18,8 & 17,5 & 15,6 & 14,7 \\
\hline Exportations & & & & & \\
Total UMA & 7,9 & 6,5 & 7,1 & 6,1 & 5,9 \\
Europe & 66,7 & 67,9 & 67,0 & 67,8 & 66,1 \\
Amérique & 14,5 & 6,2 & 6,3 & 5,9 & 6,1 \\
Asie & 18,6 & 16,1 & 15,7 & 17,5 & 18,8 \\
\hline
\end{tabular}

Source : à partir de Bank al Maghrib, rapport annuel 1996.

Il apparaît aussi que, contrairement aux pays d'Amérique Latine dans leurs relations avec les Etats-Unis, les économies de la zone sud ont une structure du commerce extérieur fortement polarisée sur l'UE. Cette dernière a représenté en 1993 plus de $49 \%$ de leurs exportations et plus de $52 \%$ de leurs importations. En 1993, les exportations vers la 
Communauté Européenne ont un indice d'intensité relative égal à 1,9 ; il s'élève à 2,0 pour les importations en provenance de la $\mathrm{CE}^{\mathrm{iii}}$. Ces données confirment l'attraction commerciale exercée par la zone Europe. Cette concentration géographique du commerce international est loin d'être similaire pour tous les pays de la zone méditerranéenne. En effet, les pays du Maghreb se caractérisent pas une concentration particulièrement forte de leurs échanges avec l'UE qui contraste avec les autres pays. Ainsi, les échanges du Maroc avec la CE ont évolué de 10,6 \% du PIB en 1970 à 15,2 \% en 1993 ; ceux de la Tunisie de 10,8 \% à 23,9\% alors que les échanges de la Turquie sont passés sur la même période de 2,2\% du PIB à 6,1\%. En outre, les échanges commerciaux entre l'UE et la zone Méditerranée sont asymétriques. En effet, si l'UE est très importante pour la Méditerranée, il n'en va de même - du moins quantitativement - de la seconde pour la première. Les pays méditerranéens n'ont représenté en 1993 que 8,6\% des exportations de l'UE et 5,8\% des ses importations. L'importance de cette zone pour l'UE réside dans le fait que c'est là quelle réalise ses excédents commerciaux les plus élevés.

Si la régionalisation n'est pas susceptible de servir de base à l'intégration des économies sud-méditerranéennes à l'économie mondiale, il convient de s'interroger sur l'état de leur insertion internationale.

\section{Difficultés de l'insertion mondiale}

Elle s'est fortement dégradée durant les décennies 70 et 80 . Deux facteurs ont joué dans ce processus : une détérioration des termes de l'échange avec le retournement du prix des matières premières d'une part et, les plans d'ajustement, suite la crise de la dette extérieure d'autre part. Il convient cependant de relativiser ces difficultés d'insertion. En effet, les pays de la zone Afrique du Nord ont opéré, au cours des années 80 et 90, une transformation importante de la structure de leurs exportations marquée par la progression de la part des produits manufacturés et le déclin des produits primaires. Ainsi, les exportations manufacturières du Maroc sont passées de 11,6 \% des exportations totales en 1980 à 23,2 \% en 1994. En termes de PIB, les exportations de produits manufacturés ont représenté $5 \%$ du PIB en 1994 contre seulement $2 \%$ en 1980 (World Bank, 1995a). Pour la Tunisie, les exportations de produits manufacturés de 42,2 \% en 1987, ont atteint $53 \%$ en 1994.

Ces tendances se traduisent par une réduction significative du déficit au niveau du solde manufacturé qui est passé de près de $18 \%$ du PIB en 1978 à près de $10 \%$ en 1992 pour la Tunisie ; et pour le Maroc respectivement de $15 \%$ du PIB à près de 7,5 \% (Bensidoun et Chevallier, 1996). Si la crise de la dette a joué un rôle dans ce mouvement, avec la réduction de la demande interne, il n'en demeure pas moins que cette évolution des soldes manufacturés traduit une amélioration significative de l'insertion de ces pays, puisqu'elle apparaît à un moment où le solde des produits primaires se dégrade. En conséquence, ces pays sont désormais susceptibles de s'engager dans une nouvelle dynamique de croissance tirée par l'extérieur. Cependant, la Tunisie et le Maroc ont une concentration très importante de leurs 
exportations manufacturières. Leur base industrielle demeure donc étroite, car fortement polarisée sur quelques produits clés intensifs en main-d'œuvre peu qualifiée. Cette situation contraste avec celle d'autres pays de la zone méditerranéenne, mais aussi avec celle des PECO, potentiellement concurrents du point de vue du processus d'intégration régionale en cours en Europe. Une telle concentration interdit à ces pays de confronter directement leur industrie nationale au marché mondial, comme les pays d'Asie du Sud-Est ont pu le faire depuis les années $70^{\text {iv }}$. De plus, ces expériences asiatiques se sont appuyées au départ sur un interventionnisme important de l'Etat qui ne semble plus de mise aujourd'hui.

\section{Des comptes courants structurellement déficitaires}

Le graphique 1 fait apparaître une dégradation générale du compte courant du Maroc et de la Tunisie depuis 1988.

Graphique 1 Solde du compte courant du Maroc et de la Tunisie. 1988 - 1996. En millions de dollars.

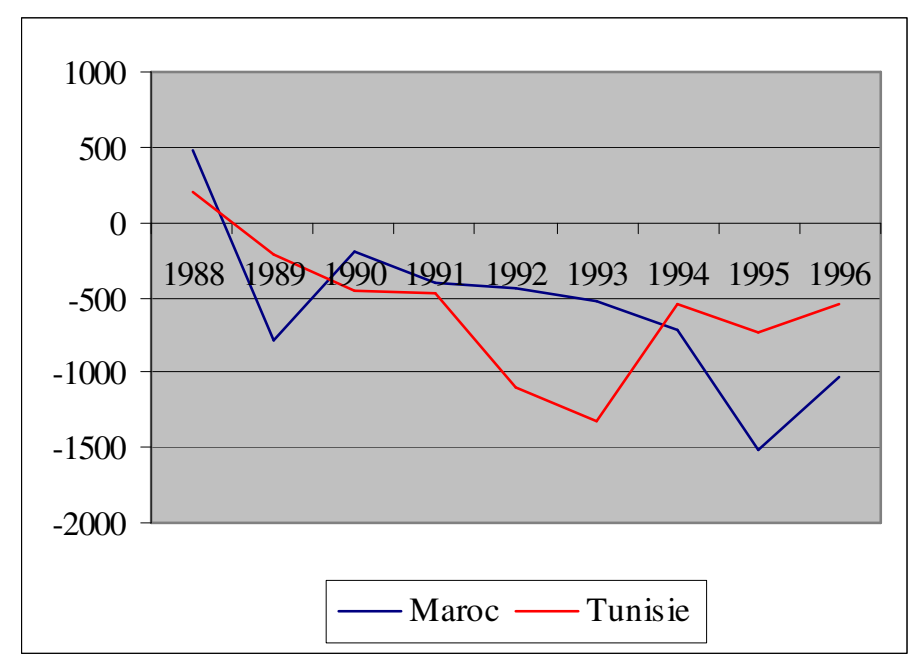

Source : Fonds Monétaire International

La dégradation de la balance commerciale des deux économies apparaît comme l'une des causes essentielles de l'aggravation de leur déséquilibre extérieur. Le déficit commercial du Maroc s'est élevé en 1996 à 2259 millions de dollars contre 1697 millions en 1989, mais, rapporté $\mathrm{PIB}$, ce déficit commercial est stable à près de 7,4 \% du PIB. Le solde commercial de la Tunisie est passé quant à lui d'un déficit de 1207 millions de dollars en 1989 (12\% du PIB) à un déficit de 1800 millions en 1996 (9,2\% du PIB). En outre, du point de vue du financement de ces déséquilibres, les réserves du Maroc représentaient en 1996 3,2 mois d'importations et celles de la Tunisie 2,5 mois. Cependant, il convient de relever que la dégradation générale de la situation des comptes extérieurs du Maroc est plus préoccupante que celle de la Tunisie. En effet, en termes de mois d'importations, en 1994, les réserves marocaines représentaient encore 4,8 mois. Depuis cette année, cet indicateur s'est dégradé régulièrement alors que l'on observe une situation inverse pour la Tunisie dans la mesure où, 
en 1990, les réserves représentaient 1,7 mois d'importations. Cette différence doit être rapprochée de l'appréciation du taux de change effectif réel du dirham beaucoup plus forte que celle du dinar (graphique 2). Selon la Banque Mondiale (World Bank 1995a), cette appréciation explique la réduction de deux points de pourcentage des exportations dans le PIB du Maroc.

Graphique 2 Taux de change effectif réel du dirham et du dinar. Base $100=1990$.

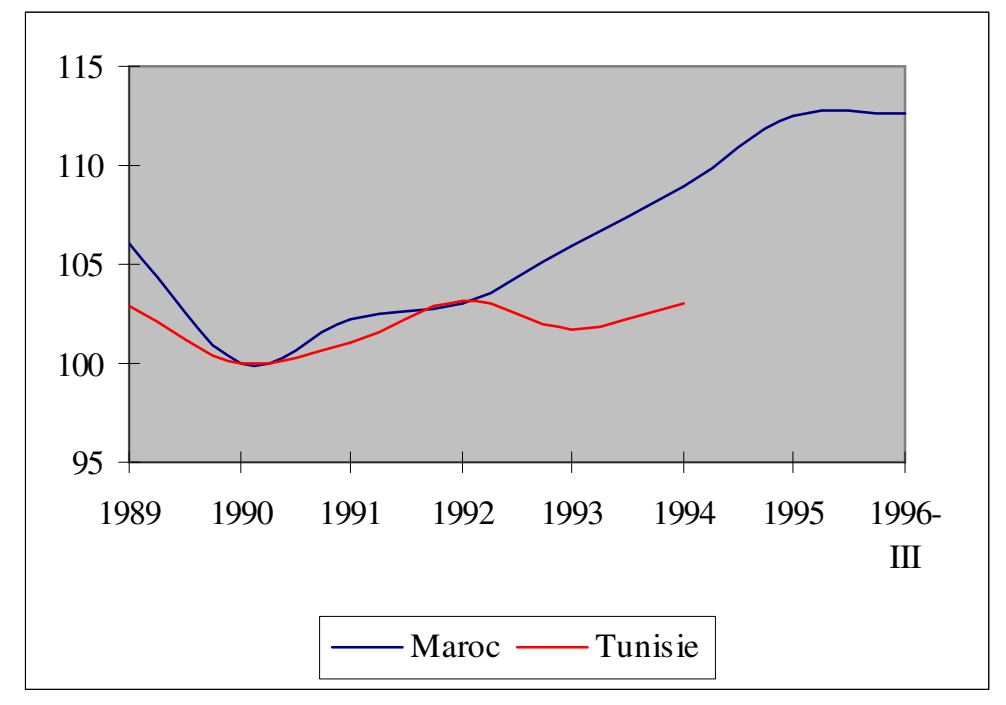

Source : Fonds Monétaires International pour le Maroc et Banque Mondiale pour la Tunisie

La dégradation des comptes extérieurs s'est effectuée dans un contexte d'ouverture croissante sur l'extérieur : le taux d'ouverture du Maroc est passé de 15,9\% en 1970 à 24,9\% en 1995 ; celui de la Tunisie de $16,9 \%$ à 35,9 \% au cours de la même période. La poursuite $\mathrm{du}$ processus d'ouverture, qui devrait se traduire par un abaissement significatif du taux effectif de protection, et la nécessité d'importer des biens d'équipement nécessaire au développement, laissent présager une situation durable de déficits courants. En effet, les autres postes du compte courant du Maroc et de la Tunisie ne sont pas en mesure de compenser les déséquilibres commerciaux. Les revenus d'investissement, reflets de l'importation de capital, sont et resteront déficitaires. Ainsi, le poste revenu de la balance des paiements a été déficitaire en moyenne annuelle de 1131 millions de dollars pour le Maroc et de 551 millions pour la Tunisie sur la période 1988 - 1995. Si les transferts des revenus des travailleurs maghrébins à l'étranger demeurent importants en termes relatifs dans les deux économies - en moyenne près de 7 \% du PIB du Maroc entre 1989 et 1994 - ils sont insuffisants en valeur absolue pour couvrir les déficits des autres postes. Ces transferts ont été en moyenne annuelle, sur la période 1988 - 1995, de 1812 millions de dollars, pour le Maroc, et de 539 millions, pour la Tunisie. Surtout, ils pourraient être appelés à devenir moins prépondérants au fur et à mesure du processus d'intégration des populations dans les 
économies européennes. On note déjà au Maroc une relative stagnation de ces transferts depuis 1990.

C'est face à ce constat de blocage, à cette situation de "vide stratégique " en termes d'insertion dans l'économie mondiale que l'idée de " sas " s'impose. Dans cette perspective, les pays sud-méditerranéens pourraient entrer dans un double processus - de mondialisation et d'intégration régionale - par l'intermédiaire de l'Europe.

\section{B. L'articulation des balances des paiements entre l'Europe et le Sud de la Méditerranée : une dynamique de développement}

L'analyse prospective de la balance des paiements de l'UE vis-à-vis des pays du Maghreb fait apparaître un excédent courant significatif, dégageant un surplus d'épargne qui pourrait servir au financement du développement de la rive Sud.

Compte tenu de l'intensité des flux réels entre les deux rives, peut-on envisager une articulation cohérente entre l'excédent courant européen - porteur d'épargne pouvant s'investir à l'extérieur de la zone - et le déficit sud-méditerranéen synonyme de besoins de financement?

D'une manière générale, l'intégration financière internationale, en raison de l'importance des flux de capitaux qu'elle sous-tend, allège les contraintes d'équilibre des comptes extérieurs. Elle peut ainsi permettre à des économies en développement d'importer les biens d'équipement, source de gains de compétitivité futurs et d'une capacité de remboursement favorable au rééquilibrage des comptes extérieurs. Cela signifie que le déficit du compte courant n'est pas en soit le signe d'une mauvaise santé économique, il peut être aussi le signe d'un développement rapide.

Cependant, deux conditions nous paraissent devoir être réunies pour qu'une dynamique financière durable puisse se développer: une amélioration de la structure des entrées de capitaux et la pérennité d'un excédent de l'épargne européenne.

\section{Une amélioration de la structure des entrées de capitaux}

$\mathrm{Si}$, d'une manière générale, la part des financements extérieurs privés en $\%$ de la FBCF au Maroc et en Tunisie est proche de celle d'autres pays émergents ${ }^{v}$, il n'en demeure pas moins que les relations financières avec l'extérieur doivent connaître d'importantes mutations par rapport à la situation actuelle pour accroître le volume de ces flux privés. En effet, l'insertion internationale des pays du Sud de la Méditerranée sur le plan financier reste marquée par la prépondérance des apports publics dans le total des financements extérieurs. Sur la période 1978 - 1992, la part des apports publics CAD dans les apports financiers nets a été de plus de $60 \%$ pour la zone Méditerranée (Algérie, Maroc, Tunisie, Turquie, Egypte et Israël) contre $50 \%$ pour l'ensemble des PED.

Cette prépondérance des financements extérieurs publics illustre l'ampleur de la tâche à accomplir pour réaliser l'intégration financière internationale de ces économies. En effet, compte tenu des limites budgétaires atteintes dans la plupart des pays industriels, seuls 
les marchés de capitaux privés sont susceptibles d'assurer des financements compatibles avec les volumes exigés par le développement économique de la région ${ }^{\mathrm{vi}}$.

L'analyse de la composition du compte financier au niveau des entrées de capitaux fournit des éléments tout à fait intéressants au niveau des flux privés.

Tableau 2 Composition des entrées de capitaux au Maroc et en Tunisie, 1988 - 1995. En millions de dollars

\begin{tabular}{|l|c|c|c|c|c|c|c|c|}
\hline \multicolumn{1}{|c|}{ MAROC } & 1988 & 1989 & 1990 & 1991 & 1992 & 1993 & 1994 & 1995 \\
\hline Investis. directs & 85 & 167 & 165 & 317 & 422 & 491 & 551 & 290 \\
\hline Investis. de portefeuille & & & & 2 & 1 & 24 & 238 & 20 \\
\hline Autres investissements & 65 & 749 & 1991 & 1082 & 851 & 480 & 144 & -366 \\
\hline TOTAL & 150 & 916 & 2156 & 1401 & 1274 & 995 & 933 & -56 \\
\hline IDE/ PIB en \% & & 0,7 & 0,6 & 1,1 & 1,5 & 1,8 & 1,8 & 0,9 \\
\hline \multicolumn{1}{|c|}{ TUNISIE } & & & & & & & & \\
\hline Investis. directs & 61 & 78 & 76 & 125 & 526 & 562 & 432 & 264 \\
\hline Investis. de portefeuille & 8 & 19 & 3 & 36 & 50 & 24 & 16 & 23 \\
\hline Autres investissements & 201 & 164 & 589 & 506 & 758 & 836 & 1029 & 990 \\
\hline TOTAL & 270 & 261 & 668 & 667 & 1334 & 1422 & 1477 & 1277 \\
\hline IDE/ PIB en \% & & 0,8 & 0,6 & 1 & 3,4 & 2,8 & 1,5 & 1,5 \\
\hline
\end{tabular}

Source : Fonds Monétaire International

Les investissements directs étrangers (IDE) dans les deux économies considérées sont un élément particulièrement important du compte financier. En effet, le processus de mutations économique et financière engagé après 1988 a favorisé un accroissement régulier des entrées de capitaux au titre des investissements directs. En raisonnant en pourcentage de PIB, ces investissements directs exercent un impact relatif plus important que ne le laissent apparaître les montants absolus qui demeurent faibles par rapport à nombre de pays émergents d'Amérique Latine et d'Asie de l'Est. Or, ces investissements sont un moteur particulièrement important de l'insertion internationale d'une économie. Surtout, les investissements directs ont une moindre volatilité que les investissements de portefeuille. La faiblesse de ces derniers en Tunisie et au Maroc est ainsi un élément de stabilité économique. Cependant, cet avantage ne doit pas cacher le fait que cette faiblesse des investissements de portefeuille illustre les difficultés des économies sud-méditerranéennes à développer un marché financier apte à s'intégrer dans la finance internationale, avec pour conséquence une tendance à la dégradation du solde global de la balance des paiements au Maroc et, dans une moindre mesure, en Tunisie, au cours de la période 1988 - 1995. Ainsi, le Maroc est passé d'un excédent de 264 millions de dollars en 1988 à un déficit de 763 millions en 1995. Le solde global tunisien est plus difficile à interpréter en raison de la réalisation de grands travaux au début des années 90 qui ont artificiellement dégradé les comptes extérieurs de l'économie. Plus précisément, la dégradation du solde global a été régulière sur la période 1988 - 1992, puis la situation s'est quelque peu améliorée. 


\section{Les conditions de la pérennité d'un excédent européen d'épargne}

Artus (1997) rappelle que la tendance à un excédent des comptes courants de l'UE, qui s'accentue depuis 1993, répond à des facteurs structurels dont l'impact sera long à se résorber : le vieillissement de la population qui pousse à la hausse le taux d'épargne, une discipline budgétaire (pacte de stabilité et de croissance) qui rend peu probable une dégradation de la balance courante européenne par la désépargne publique, un écart structurel de croissance tendancielle favorable au reste du monde qui fait que les importations européennes croîtront à un rythme inférieur aux exportations, enfin une inflation qui devrait rester durablement à un bas niveau. Cette structure excédentaire est encore plus nette vis-à-vis du Maghreb.

Cependant, l'évolution de la dynamique financière avec les pays de la Méditerranée est conditionnée par celle de l'épargne dans l'UE et par les besoins concurrents susceptibles de se manifester à l'Est de l'Europe qui pourraient engendrer une pénurie d'épargne néfaste à la formation d'un espace financier euro-méditerranéen. L'expérience de la réunification de l'Allemagne a montré comment des transferts d'épargne de l'ouest à l'est ont engendré un recul de l'excédent du compte courant allemand, synonyme de moindre capacité à exporter des capitaux. Cet argument peut être nuancé par le fait que le choc de la réunification allemande a été ample et rapide à produire ses effets en raison de l'unification politique, situation très spécifique par rapport à celle d'autres pays est européens.

La situation de la balance des paiements de l'Union européenne et des économies sud-méditerranéennes paraît ainsi favorable à l'intensification des flux financiers entre les deux rives. L'intensification des échanges commerciaux pourrait permettre au surplus d'épargne formé, au Nord, de financer le développement du Sud, constituant ainsi un espace financier euro-méditerranéen. Les pays du Maghreb qui ont une place privilégiée dans les échanges avec l'UE - relativement aux autres pays méditerranéens - sont ainsi particulièrement concernés par les effets systémiques régionaux de la mise en place d'un espace monétaire unique en Europe.

\section{L'impact de l'euro sur les systèmes financiers sud méditerranéens}

Dans la perspective d'une meilleure intégration internationale, la plupart des pays en développement ont été conduits à appuyer le processus d'ouverture sur une libéralisation de leur système financier. C'est le cas du Maroc, de la Tunisie et dans une certaine mesure de l'Algérie. Compte tenu de l'intensité de leurs relations avec l'UE, il est indispensable d'envisager l'impact que la nouvelle donne financière et monétaire liée au passage à la monnaie unique aura sur les systèmes financiers de ces pays. 


\section{A. L'euro, créateur de nouveaux modes de relations financières et monétaires}

La mise en place de l'euro semble devoir enclencher de profondes mutations dans l'ajustement international des portefeuilles et les mouvements de capitaux, débouchant sur un véritable marché financier européen susceptible de fournir aux pays tiers des conditions favorables pour se procurer des capitaux. L'euro, unité stable de libellé des actifs financiers pourrait aussi affirmer son rôle de réserve de valeur et développer sa dimension internationale, en particulier d'ancrage de monnaies tierces.

\section{L'euro et le marché financier européen}

Les marchés financiers européens se caractérisent encore actuellement par une relative segmentation. L'introduction de l'euro s'interprète comme le catalyseur d'un processus d'intégration financière puisque la transparence créée par l'unification de l'unité de compte fera ressortir les segmentations d'ordre réglementaire et fiscal qui tendront alors à s'atténuer. Comme le soulignent Prati et Schinasi (1997), l'euro doit créer le plus important marché financier domestique du monde. Sur la base d'une union à quinze et des chiffres de 1995, les actions, obligations et actifs bancaires représenteraient 27000 milliard de dollars, soit 94 \% du PIB mondial, contre 24000 milliards pour l'Amérique du Nord.

D'abord, les coûts de transactions seront réduits avec l'élimination du change intra européen, la standardisation des instruments financiers et la concurrence renforcée entre intermédiaires sur un marché élargi. Ensuite, l'épargne sera attirée par la transparence accrue du fait de l'atténuation progressive des spécificités locales avec l'uniformisation des pratiques financières entraînée par la concurrence. De plus se produira un mouvement d'européanistion des portefeuilles des investisseurs institutionnels soumis à la règle de congruence, les obligeant à adosser leurs engagements en devises sur des avoirs dans la même devise, et qui acquerront alors indifféremment des actifs financiers en euro dans un espace monétairement unifié.

Le décloisonnement des marchés engendrera une externalité positive fondée sur l'augmentation de la liquidité qui pourra conduire les investisseurs américains ou japonais, qui ne détiennent que $7 \%$ de valeurs européennes dans leur portefeuille, à reconsidérer leur position face à un marché plus profond et plus large. En outre, l'attraction exercée par l'Europe devrait être accrue si l'on considère la volatilité des rendements des marchés de titres. Celle-ci semble liée à la volatilité des changes (Prati, Schinasi, 1997). Ainsi dans la mesure où l'Union Economique et Monétaire sera une zone monétaire unifiée, on peut présumer que la volatilité des cours des obligations et des actions s'y réduira de façon substantielle. En conséquence, le marché financier européen de par sa plus grande stabilité devrait attirer les investisseurs non européens.

L'euro ne sera pas seulement une monnaie de placement, mais aussi une monnaie d'endettement (McCauley, White, 1997. La transformation des marchés européens induite par 
l'euro et le caractère excédentaire de la zone, en termes de paiements courants, semblent aussi devoir faire de celle-ci un prêteur net vis-à-vis du reste du monde.

L'attrait du marché européen pour satisfaire les besoins de financement des tiers passera d'abord par le marché obligataire large et profond avec des coûts de transaction réduits et un risque de change potentiellement plus limité que sur les anciennes devises européennes. Cette demande de capitaux ne devrait pas exercer de tension à la hausse des taux d'intérêt compte tenu de l'excédent structurel des paiements courants et de l'attractivité de la zone pour les investisseurs non européens. La Banque Centrale Européenne pourrait ainsi viser son objectif de stabilité des prix avec des taux d'intérêt bas ${ }^{\mathrm{vii}}$.

L'internationalisation de l'euro, tant du côté des placements que des emprunts, pourrait ainsi faire du marché financier européen un instrument de réallocation des flux internationaux de capitaux, constituant pour les pays du sud de la Méditerranée fortement demandeurs un réservoir de fonds mobilisables à des coûts relativement modérés. Cette relation financière, constitutive de l'espace financier euro-méditerranéen, débouche sur la question de l'ancrage des monnaies du sud à l'euro.

\section{L'ancrage des monnaies du sud à l'euro}

Cet ancrage peut entraîner des effets de synergie extrêmement importants du point de vue de la formation d'un espace euro-méditerranéen. Il aurait pour avantage d'intensifier les externalités positives engendrées par l'utilisation internationale d'une monnaie. Ces synergies reposent sur les économies d'échelle (Alogoskoufis et Portes, 1991) engendrées par l'utilisation internationale d'une monnaie. Plus une monnaie est utilisée, plus les acteurs ont intérêt à l'utiliser.

La difficulté pour les pays du Maghreb est de déterminer les conditions dans lesquelles un tel ancrage sur la monnaie européenne peut se réaliser. D’une manière générale, il est important de considérer que l'ancrage à une monnaie n'est pas synonyme de définition de parité fixe vis-à-vis de la devise dominante. De ce point de vue, une stratégie d'ancrage n'implique pas nécessairement le renoncement à toute souplesse en matière de politique de change pour les autorités monétaires. En outre, les stratégies d'ancrage peuvent répondre à deux logiques différentes.

D'abord, les autorités monétaires peuvent chercher à stabiliser le taux de change nominal de leur monnaie en niveau ou en termes de régularité dans ses variations à court terme : c'est l'ancrage nominal. Une telle stratégie est généralement suivie par les pays qui cherchent à importer la crédibilité de banques centrales plus vertueuses en matière de stabilité des prix. De nombreux pays d'Amérique Latine ont, dans les années 80, adopté cette stratégie d'ancrage nominal dans le cadre de leur plan de stabilisation.

La seconde logique recherche la stabilité à long terme du taux de change réel en mettant l'accent sur la compétitivité de l'économie : c'est l'ancrage réel. Cette stratégie a été 
suivie par les pays d'Asie de l'Est et du Sud-Est, la politique de change étant alors partie prenante de leur stratégie d'intégration à l'économie mondiale.

Quelle peut être la stratégie suivie par les pays du Maghreb ? Le bilan des expériences des pays d'Amérique Latine et d'Asie permet d'esquisser quelques éléments de réponses. Celles-ci montrent les difficultés de l'ancrage, mais pour des raisons assez différentes selon les pays. Ainsi, les pays d'Amérique Latine se sont heurtés aux limites inhérentes à la stratégie des plans de stabilisation : la forte appréciation du taux de change réel est rapidement apparue contradictoire avec le maintien de la compétitivité de l'économie domestique. Cette appréciation trouve en partie ses origines dans le fait que le rythme d'inflation de ces pays a souvent été supérieur à celui de l'inflation du pays ancre, et ce, alors même que les autorités monétaires répugnent à modifier le taux de change nominal dans le sens de la dépréciation puisque la rigidification de l'ancrage est interprétée comme le gage de la crédibilité du plan de stabilisation. Les pays d'Asie ont eu d'importantes difficultés à mettre en cohérence leur stratégie d'ancrage avec les mutations économiques qui ont affecté la zone depuis le début des années 80. En effet, l'importance historique du commerce avec les Etats-Unis et l'absence d'incitation de ces pays à coordonner leur politique monétaire (Bénassy-Quéré, 1997) les ont conduit à ancrer leur monnaie de manière implicite ou explicite au dollar. Or, les mutations structurelles de la zone au cours des années 80 ont fragilisé cette stratégie (Ito et alii, 1996).

Le régime de change du Maroc se caractérise par un rattachement de la monnaie domestique à un panier spécifique composé de plusieurs devises. L'avantage d'une telle stratégie est d'amortir les conséquences à la fois l'appréciation et la dépréciation des devises pour la monnaie domestique si le panier est composée de devises représentatives des paiements extérieurs de l'économie considérée. Cette composition doit donc évoluer au cours du temps au fur et à mesure des transformations des relations économiques extérieures. Ainsi, selon Idrissi (1998), le panier a été modifié à quatre reprises depuis sa création dans les années 70 dans le sens d'une réduction de la part du dollar et de l'accroissement de celles du franc français et du mark. Le franc suisse a quant à lui disparu du panier. Depuis la fin des années 80, les autorités ont progressivement libéralisé le contrôle des changes. La gestion des devises a ainsi été décentralisée. En 1993, la convertibilité du dirham, au titre des transactions courantes, a été instaurée et en 1996 un marché des changes a été créé. Cependant, le panier demeure la référence pour la cotation de la monnaie marocaine. Un avantage souvent avancé de cette stratégie est la stabilité ainsi obtenue du taux de change du dirham depuis les années 80. Cependant, comme nous l'avons déjà souligné, cette apparente stabilité ne doit pas cacher la tendance du dirham à s'apprécier en termes réels depuis le début des années 90, ce qui ne peut que peser sur la croissance économique du pays. De même, la présence du dollar dans le panier est souvent justifiée par l'importance des exportations de phosphate pour l'économie, exportations qui sont libellées dans cette devise. Or, on observe depuis 1991 un recul du poids 
des phosphates dans les exportations : de $23 \%$ en 1980 à 4,5\% en 1994 (World Bank, 1995a). A contrario, plusieurs indicateurs tendent à montrer que l'ancrage du dirham à l'euro pourrait se révéler être une stratégie cohérente du point de vue de l'insertion internationale du Maroc :

- la composition géographique du déficit commercial du Maroc en 1995 montre une prépondérance de l'Union européenne (près de $50 \%$ du déficit total) ;

- la composition en devises de la dette extérieure à long terme met en avant un recul de la part du dollar entre 1980 et 1995 au profit d'un rééquilibrage en faveur des monnaies européennes. Plus précisément, si l'on tient compte de la composition estimée des emprunts en devises convertibles - qui ont connu une croissance rapide sur la période - Idrissi (1998) estime que pour l'année 1995 la part du dollar dans la dette totale est de $39 \%$ et celle des monnaies européennes de $36 \%$;

- le commerce extérieur du Maroc, nous l'avons vu, est fortement polarisé vers l'Union européenne avec, depuis 1991, un recul des exportations vers cette zone de produits primaires qui peuvent être en partie libellées en dollars.

La Tunisie a adopté depuis 1978 un régime de change à flottement dirigé. Les calculs de volatilité tendent à montrer que le dinar est déjà fortement ancré, de manière implicite, sur les monnaies européennes (Mouley, 1998) ${ }^{\text {viii }}$. En outre, on observe dans la composition en devises des dépenses une prépondérance des monnaies européennes (près de $43 \%$ en 1995 pour le franc français, le deutschemark, la lire et la livre) ; leur part étant de $29 \%$ environ pour les recettes.

Au total, l'expérience des pays asiatiques et le degré d'intégration commerciale et financière entre le Mahgreb et l'Union européenne mettent ainsi en avant le fait qu'un ancrage à l'euro aurait pour avantage d'une part, de stabiliser la valeur en monnaie domestique de la dette extérieure libellée en euro dont l'importance devrait s'accroître et, d'autre part, de stabiliser les monnaies domestiques vis-à-vis de la monnaie du principal partenaire commercial. Une telle stratégie favoriserait aussi les flux d'investissements directs étrangers en provenance de la zone euro et de conduirait à une uniformisation progressive des pratiques monétaires.

Cependant, comme nous l'avons déjà souligné, une stratégie d'ancrage ne doit pas être confondue avec l'élaboration d'un régime de change rigide. Les économies du Maghreb doivent en effet pouvoir utiliser le taux de change pour faire face aux ajustements nécessaires liés au développement économique.

\section{B. Des systèmes financiers adaptés}

A l'avenir, seuls les capitaux privés seront susceptibles d'assurer les financements compatibles avec les volumes exigés par le développement économique de la région, capitaux importés, mais aussi capitaux issus d'une épargne intérieure qui est loin d'être négligeable en Afrique du Nord. Dans les deux cas, se pose le problème de l'efficacité du système financier 
apte d'une part à attirer les investisseurs et d'autre part à soutenir les promoteurs de projets facteurs de développement. Un tel système doit être à la fois un système de marché et un système intermédié.

\section{Système de marché}

La globalisation a profondément modifié les modalités de financement de l'économie mondiale et par la même l'accès des économies nationales aux ressources : les financements par titres représentent le compartiment le plus dynamique du système financier international. Or ce qui caractérise la finance de marché, ce sont des comportements d'évaluation des opportunités de financement largement standardisés tant dans les pays développés que dans les pays en développement qualifiés aujourd'hui de pays émergents. Le mouvement de montée du rôle des valeurs mobilières ou titres négociables, qualifié de mobiliérisation, concerne le système financier des pays du sud, aussi bien avec la mise aux normes internationales de leurs Bourses de valeurs accompagnant les politiques de privatisation et d'ouverture du capital des sociétés aux non-résidents ${ }^{\mathrm{ix}}$, qu'avec les premières émissions sur les marchés internationaux de titres de sociétés privées ${ }^{\mathrm{x}}$.

Mais il ne faudrait pas surestimer l'aspect quantitatif de la contribution de la Bourse au financement de l'économie. Au Maroc, par exemple, la capitalisation boursière a fortement progressé de 1990 à 1996, passant de 3,6 \% à 23,6 \% du PIB ; pourtant sur la même période, les augmentations de capital en numéraire, très volatiles, sont passées de $2,16 \%$ à moins de $1 \%$ de la formation brute de capital fixe. De plus, si l'on déduit les distributions de dividende, on sait que même, en France, l'apport net de fonds aux sociétés par les actionnaires demeure modeste.

Pourtant la réforme boursière se justifie parfaitement, d'un point de vue qualitatif, si elle s'accompagne d'une modification du comportement des entreprises. Comme le remarque Fry (1997) en matière bancaire, la libéralisation financière nécessite des conditions préalables pour réussir ; il en va de même en matière boursière. Suite au sommet du G7 de Lyon de juin 1996, un rapport du groupe des 10 et de représentants d'économies de marchés émergents (Group of Ten, 1997) présentant les conditions de stabilité et de consolidation des systèmes financiers insiste particulièrement sur l'accès à l'information et la transparence. L'accroissement du nombre de sociétés cotées est un excellent indicateur du développement d'une allocation des ressources soumises aux contraintes du marché. Et sur ce point beaucoup reste à faire dans les pays du Maghreb.

Paradoxalement, les bons résultats boursiers des dernières années ${ }^{\mathrm{xi}}$ contrastent avec la lenteur de la diffusion du rôle du marché qui découle de la stagnation du nombre de sociétés acceptant ses règles. En 1997 on ne comptait que 48 sociétés cotées à Casablanca ${ }^{\text {xii }}$ et 30 à Tunis. L'envolée des cours, principale cause de la croissance de la capitalisation, avec les privatisations, reflète souvent un manque de papier. Malgré un coût élevé du crédit, les entreprises hésitent à solliciter les marchés : l'autofinancement et le crédit bancaire à court 
terme restent très largement prédominants xiii . Les institutions financières sont sur-représentées, dans la cote, par rapport aux sociétés industrielles ou de services. Si les privatisations sont la principale source d'approvisionnement de la cote, de belles entreprises privées semblent réticentes à l'entrée en Bourse. Le renforcement des exigences en matière d'information émanant des sociétés cotées qui va de pair avec la mise aux normes internationales, indispensable à l'ouverture aux marchés de capitaux internationaux, se heurte à une culture de discrétion liée à la structure familiale du capital et à des réflexes de protection fiscale.

Au fond, dans les pays en développement, l'important est moins la mobiliérisation en tant que telle, que la marchéisation qu'elle entraîne. La marchéisation est le processus d'accroissement du rôle des mécanismes de marché dans l'allocation des ressources. Cela va bien au-delà d'une seule négociabilité des actifs, impliquée dans la mobiliérisation, pour recouvrir la diffusion à l'ensemble du système financier de la concurrence dans la détermination des prix, des taux d'intérêt, que les actifs soient ou non des titres négociables sur un marché secondaire. La marchéisation concerne aussi bien les fonds propres des sociétés non cotées, capital-investissement ou capital-risque au sens large, secteur qui pourrait être vivifié par les investissements directs étrangers, que les circuits bancaires où la mutation n'a pas encore atteint son terme. Une telle situation est instable, dans la mesure où l'indispensable poursuite de la libéralisation et l'irruption de la concurrence des banques étrangères, du fait des accords de l'OMC et de l'accord d'association avec l'Union européenne, devraient conduire à de profondes restructurations.

\section{Un système intermédié}

Il convient d'être prudent face à cette présentation souvent développée selon laquelle la libéralisation financière s'assimilerait à une désintermédiation au sens de passage à une structure de financement direct. D'abord, les titres de marché entrent le plus souvent dans un processus de financement indirect, d'intermédiation de bilan, dans la mesure où ils sont émis ou détenus par des intermédiaires financiers (banques, fonds de pension, compagnies d'assurance, fonds d'investissement). La mobiliérisation est tout à fait compatible avec le financement indirect. Ensuite, comme le souligne Fry (1997), dans les pays en développement, les systèmes financiers restent dominés par les banques commerciales. Cette structure, largement intermédiée, n'est pas en tant que telle un obstacle à la libéralisation financière. L'enjeu de celle-ci libéralisation financière est bien la marchéisation des banques, c'est à dire leur banalisation dans l'économie de marché pour en faire des entreprises comme les autres, soumises à la concurrence, tant pour la détermination du prix de leurs services, que pour leurs conditions de financement.

$\mathrm{Au}$ Maroc et en Tunisie, le processus de réforme semble fonctionner par à-coups. Alors que le système bancaire était fortement contraint dans son activité par le cloisonnement des statuts, l'administration des taux d'intérêt et l'orientation forcée d'une partie de ses 
emplois vers le Trésor ou des secteurs prioritaires, les lois bancaire de novembre 1993, au Maroc, et de février 1994, en Tunisie, consacrent la libéralisation des banques. Ensuite, une large avancée a concerné la Bourse, mais la réforme des autres parties du système financier semble marquer un peu le pas. Suite aux lois bancaires et au principe de déréglementation, les banques n'ont pas connu de véritable mutation. En effet, l'orientation vers les financements courts demeure largement prépondérante et la libéralisation des taux d'intérêt administrés ne semble pas avoir ouvert la voie à une large concurrence bancaire ${ }^{\mathrm{xiv}}$. Les marchés de dettes sont encore très peu développés, hormis le compartiment des titres d'Etat qui exerce d'ailleurs un effet d'éviction, malgré la réduction des emplois obligatoires, du fait de taux d'intérêt relativement élevés. Faute d'une véritable ouverture du marché des titres de créance, le système détourne l'épargne vers les dépôts bancaires utilisés au financement de crédits courts aux entreprises et de titres publics longs. Les entreprises peu enclines à donner l'information qu'exige le marché, pour effectuer par exemple une notation, y retrouvent leur compte. On rencontre ainsi un risque de blocage unissant entreprises, banques et administrations, au détriment d'une meilleure allocation des ressources et d'un accroissement du financement de l'économie par apport de capitaux étrangers

Des conditions préalables sont recommandées aux banques par les experts de la BRI, du FMI, du Groupe des 10. Elles portent sur l'adoption d'une réglementation prudentielle adéquate fondée sur une solide structure comptable et des institutions de contrôle efficaces, sur la réduction de l'implication des pouvoirs publics dans les financements et sur une amélioration des incitations des actionnaires, des dirigeants et des créanciers des banques pour développer un comportement concurrentiel de recherche de profit.

Au delà, et dans la perspective plus particulière du financement des pays de la rive sud de la Méditerranée, il faut préciser aussi les conditions de la constitution d'un espace financier euro-méditerranéen. A priori la connexion des systèmes financiers des pays en développement sur le marché n'a pas de raison de se faire plus particulièrement avec l'Europe : avec la globalisation, le marché des capitaux tend bien à être mondial. L'espace financier euro-méditerranéen ne peut se concevoir que si la densité des échanges courants entre l'UEM et la rive sud est complétée à la fois par les solidarités de change, précédemment envisagées, allant au minimum d'un libellé des opérations financières en euro, jusqu'à d'éventuels ancrages de change et par un volontarisme politique se concrétisant dans une coopération technique.

Le pragmatisme et le gradualisme dans la réforme sont nécessaires pour en minimiser les coûts et éviter tout dérapage ou gaspillage. L'acquis technique des pays européens peut encore bénéficier aux pays du sud, en particulier dans la mise en place de marchés adaptés aux sociétés moyennes ou nouvelles et de systèmes d'information performants pour éclairer les choix du plus grand nombre d'investisseurs. Les problèmes de suivi et de contrôle des opérations de marché, de règlements et de livraison ont pris une 
grande importance sur les places européennes, avec la montée des préoccupations de back office. Il n'y a pas nécessairement décalage, sur ce point, entre ces places et les marchés qui émergent, car on assiste à une irruption très rapide des techniques les plus modernes. Dans leur partenariat, les institutions financières européennes et maghrébines ne doivent pas confondre modernité et mimétisme : les produits utilisés dans les pays émergents peuvent être commercialement (front office) simples et bien adaptés aux besoins locaux, mais leur traitement, interne au système de règlement et de livraison (back office), faire appel à des techniques relativement sophistiquées.

Dans la mesure où, hormis l'Etat, les banques et quelques grandes entreprises, les agents économiques des pays en développement ne peuvent accéder directement aux marchés internationaux de titres pour se financer, on peut mettre en évidence le rôle d'intermédiaire des systèmes financiers de ces pays, intermédiaire entre le marché international des capitaux et l'économie nationale. La connexion internationale peut s'effectuer soit par les investissements directs, soit par les investissements de portefeuille.

Si l'IDE demeure, nous l'avons vu faible, l'origine de ces fonds est largement européenne, pour plus de $50 \%$, dans le cas du Maroc, en 1995, la part de la France étant la première. Pour la Tunisie, c'est l'Italie qui arrive en tête, devant les Etats-Unis et la France à égalité. Ces investissements peuvent concerner les grandes entreprises, en particulier dans le cadre des privatisations, mais il serait nécessaire qu'ils irradient aussi le PME pour leur apporter à la fois financement, technologie et méthodes de gestion. La levée des réglementations de type protectionniste sur la nationalité des entrepreneurs, les autorisations administratives et la convertibilité doit se poursuivre ${ }^{\mathrm{xv}}$. Cependant, ces flux d'IDE qui sont un facteur particulièrement important de l'insertion internationale d'une économie et dont la volatilité est limitée ne se développeront que si des structures intermédiées de capital-investissement se mettent en place. La création de fonds d'investissement et spécialement de fonds de fonds peut permettre d'associer les investisseurs européens et particulièrement les résidents maghrébins à l'étranger à des projets locaux, tout en leur assurant une certaine diversification des risques. Les techniques de mutualisation du risque et l'institution de fonds de garantie sont aussi un moyen de sécuriser l'investissement et d'attirer les capitaux extérieurs.

L'investissement de portefeuille est un canal ambigu de connexion internationale qui peut à la fois procurer d'importants moyens financiers mais qui du fait de sa volatilité soumet directement les pays en développement aux risques de crises des marchés. Les valeurs maghrébines restent encore très peu représentées dans les portefeuilles internationaux. Les récents progrès de la capitalisation ont concerné surtout les investisseurs domestiques, la part des étrangers se réduisant de $6 \%$ à $3 \%$ de 1994 à $1996^{\text {xvi }}$. La poursuite du développement du marché boursier exige l'apport de financements extérieurs. Ceux ci ne manquent pas, compte tenu de la masse des fonds gérés par les investisseurs institutionnels et de la nécessaire 
diversification de leurs portefeuilles. Mais leur accueil doit être encouragé par la poursuite de la libéralisation et l'amélioration de la qualité de l'information. La condition première de l'afflux de capitaux reste toutefois l'offre de papier par de nouvelles introductions, ce qui implique une véritable révolution dans le comportement des entrepreneurs locaux. De plus, compte tenu de la capitalisation limitée de ces nouvelles sociétés et des contraintes de gestion des investisseurs étrangers la constitution de portefeuilles collectifs proposés par des OPCVM locaux serait utile et servirait de base à la mise en place d'une industrie de la gestion sud-méditerranéeenne.

Enfin, l'investissement de portefeuille pourrait être largement sollicité s'il débouchait sur un renouveau de l'activité bancaire. Les établissements de crédit des pays du sud soumis aux contraintes du marché devraient pouvoir émettre des obligations sur le marché financier européen. Les fonds ainsi collectés seraient alors canalisés vers les multiples agents économiques promoteurs de projets de développement qui par leur taille restent tributaires du crédit. Il s'agirait de réinventer les banques de développement en finançant les investissements, mais en pratiquant le financement privé et le marché. On pourrait même imaginer des techniques de titrisation de créances bancaires, débouchant sur des fonds communs de créances proposés aux investisseurs non résidents. Ceci suppose une poursuite de la mutation des comportements des banques encore très orientées vers le court terme et peu ouvertes à la concurrence. Un relais du financement public par un financement privé soumis à l'allocation des ressources par le marché est ici crucial.

\section{Conclusion}

Les effets systémiques positifs que devraient entraîner la création de l'euro sur les pays du sud de la Méditerranée ont été soulignés. Nous avons insisté ici sur les dimensions financières de ces implications en raison de mutations très importantes que vont connaître les marchés financiers européens et des besoins de financement des économies du Maghreb. Même si la zone euro est excédentaire et donc prêteuse internationalement, les effets identifiés précédemment pourraient être atténués par différents facteurs, en particulier un dérapage de la réforme financière. En effet, si ce mouvement de réformes développant la logique de marché, dicté par la globalisation et fortement encouragé par les institutions financières internationales, ne saurait être mis en cause, tout particulièrement du fait de son effet "éducatif" en matière de comportements, il convient de s'interroger sur les choix effectués, leur utilité, leur coût. Trop souvent la réforme financière s'inspire de "produits" directement inspirés de l'expérience de pays du Nord sans analyser suffisamment quels peuvent être les besoins d'un pays émergent sur le plan financier. Seule la conjonction d'une réforme financière adaptée et de la réalisation d'une zone de relations économiques privilégiées fondées sur des échanges courants représente une chance, pour les pays du sud de participer positivement à la globalisation et de minimiser ses effets perturbateurs. 


\section{Références}

Alogoskoufis G. et Portes R. (1991), "International costs and benefits from EMU ", European Economy, Special issue, ${ }^{\circ} 1$, p.231-245.

Artus P. (1996), " A strong euro or a weak euro ?", CDC, Document de travail, n¹996-02/EI, novembre.

Artus P. (1997), " L'euro, la diversification de portefeuille et la gestion des réserves ", $C D C$, Document de travail, $\mathrm{n}^{\circ} 1997-09 / \mathrm{EI}$, septembre.

Bank al Maghrib (1996), Rapport annuel.

Bénassy-Quéré A. (1996), " Potentialities and opportunities of the euro as an international currency ", CEPII, Document de travail, n $96-09$.

Bensidoun I. et Chevallier A. (1996), Europe - Méditerranée : le pari de l'ouverture, CEPII, Economica, Paris.

Bergsten C.F. (1997), “Open regionalism ”, Institute for International Economics, Working Paper, $97-3$.

Berrigan J. et Carré H. (1997), " Exchange arrangements between the EU and countrie in Eastern Europe, the Mediterranean, and the CFA zone ", in EMU and the international monetary system, P.R. Masson, T.H. Krueger et B.G. Turtelboom (eds), IMF, Washington DC, p.122-135.

Chevallier A. (1997), "Les flux de financements extérieurs ”, Techniques Financières et Développement, $\mathrm{n}^{\circ} 47,17-20$.

El Idrissi L.S. (1998), “Dette publique et régime de change au Maroc face aux perspectives d'Union monétaire en Europe", Communication au colloque international L'euro et le financement de la croissance en Méditerranée, Marseille, Janvier.

Fry M.J. (1997), “In favour of financial liberalisation ”, Economic Journal, vol.107, May, p.754-770.

Group of Ten (1997), Report of the working party on financial stability in emerging market economies.

Gustin D. (1997), La place de l'euro dans l'économie mondiale, Mémoire pour le DEA Monnaie - Banque - Financement, Université Lumière Lyon 2.

Ito T., Isard P., Symansky S. et Bayoumi T. (1996), “Exchange rate movements and their impact on trade and investment in the APEC region ", Occasional Paper, ${ }^{\circ} 145$, IMF, Washington DC.

Jbili A., Enders K., Treichel V., Les réformes financières au Maroc et en Tunisie, Finances \& Développement, sept. 1997, p.29-31.

Krimi B. (1997), “Les enjeux de la modernisation des banques tunisiennes ", L'Economiste Maghrébin, n¹89, p.44-47.

Lawrence R.Z. (1997), "Preferential trading arrangements : the traditional and the new ", in Regional partners in global markets : limits and possibilities of the Euro-Med agreements ; A. Galal et B. Hoekman (eds), ECES et CEPR, Londres, p.13-34.

McCauley R.N. et White W.R. (1997), " The euro and European financial markets ", in EMU and the international monetary system, P.R. Masson, T.H. Krueger et B.G. Turtelboom (eds), IMF, Washington DC, p.324-388.

Mouley S. (1998), “Coûts et opportunités de l'ancrage du dinar tunisien à une zone euro en Méditerranée", Communication au colloque international L'euro et le financement de la croissance en Méditerranée, Marseille, Janvier.

Prati A. et Schinasi G.J. (1997), " European monetary union and international capital markets : structural implications and risks ", in EMU and the international monetary system, P.R. Masson, T.H. Krueger et B.G. Turtelboom (eds), IMF, Washington DC, p.263-319. 
Singh A. (1997), "Financial liberalisation, stockmarkets and economic development", Economic Journal, vol. 107, May, p.771-782.

Thygesen N. et alii (1995), International currency competition and the future role of the single European currency, Kluwer Law International, Londres.

Weiller J. (1946), Problèmes d'économie internationale, tome 1, PUF, Paris.

World Bank (1995a), Kingdom of Morocco, country economic memorandum towards higher growth and employment, September, Washington DC.

World Bank (1995b), Republic of Tunisia, towards the 21st century country economic memorandum, October, Washington DC.

\section{Notes}

*. Maître de Conférences, GATE UMR 5824 du CNRS et Université Lumière Lyon 2, 93 chemin des Mouilles 69130 Ecully ; e-mail : allegret@gate.cnrs.fr.

**. Professeur, GATE UMR 5824 du CNRS et Université Lumière Lyon 2, 93 chemin des Mouilles 69130

Ecully; e-mail : courbis@univ-lyon2.fr

i Algérie, Maroc, Tunisie, Egypte, Liban, Syrie et Jordanie.

ii Des accords d'association ont notamment été signés avec la Tunisie (12 avril 1995), Israël (28 septembre 1995), le Maroc (26 février 1996).

iii L'indicateur d'intensité relative des échanges permet de mesurer l'ampleur de l'attraction exercée par l'UE sur les pays du Maghreb (Bensidoun et Chevallier, 1996). Un tel indicateur consiste à calculer pour les exportations et pour les importations la place qu'occupe un pays dans le commerce d'un pays partenaire relativement à la place de ce dernier dans le commerce mondial. Lorsque l'indicateur est égal à 1, les flux commerciaux sont strictement proportionnels au poids des pays dans le commerce mondial, sans signification particulière en termes d'intégration régionale. Un indicateur égal à 2 révèle des flux d'échanges deux fois plus intenses que ne le justifient les seuls poids des partenaires dans le commerce mondial.

iv Bergsten (1997) rappelle ainsi que la Chine, Hong-Kong, la Corée du Sud et Taïwan (à l'instar du Japon) sont les seules puissances économiques qui n'ont pas participé à des accords régionaux avant la constitution de l'APEC. Pour l'auteur, cela traduit leur volonté d'avoir une stratégie de développement fondée sur la mondialisation.

v $20 \%$ au Maroc, $13 \%$ en Tunisie, $19 \%$ au Chili, $16 \%$ en Indonésie et $12 \%$ aux Philippines et en Argentine sur la période 1990 - 1995 d'après Chevallier (1997), p.18.

${ }^{\text {vi }}$ Cette observation doit cependant être relativisée si l'on considère que le niveau de l'épargne intérieure brute est loin d'être négligeable dans l'ensemble Afrique du Nord : 24,7 \% sur la période 1981-1989. Cela signifie que l'un des défis majeurs de ces économies est de mobiliser cette épargne intérieure en direction du financement des entreprises tout en favorisant simultanément l'accès aux marchés financiers extérieurs. La seconde partie de ce travail reviendra sur ce défi important.

vii Nous nous démarquons ainsi de McCauley et White (1997) qui, se limitant à une stricte approche en termes de portefeuille, concluent à une augmentation des taux d'intérêt sur les titres en euro en raison d'un excès d'offre de titres. Une telle éventualité est aussi retenue par Thygesen et alii (1995).

viii Une remarque similaire peut être faite pour le dirham marocain. En effet, des calculs effectués par la Banque des Règlements Internationaux montrent que la monnaie marocaine est très sensible aux fluctuations du mark par rapport au dollar.

ix Au Maroc en 1993 et en Tunisie en 1994 , la réforme boursière a institué un système à trois niveaux, avec un Conseil chargé de la réglementation, du contrôle et de la protection de l'épargne, une Bourse, à statut de société privée, et des intermédiaires de marché distincts des banques. Un système de négociation électronique devrait permettre la démultiplication de la capacité de traitement des ordres et le renforcement de la transparence et de la liquidité du marché. Un système efficace de règlement - livraison devrait aussi contribuer à la mise aux normes internationales. L'Algérie, parallèlement à sa politique de privatisation met aussi en place un tel système boursier à trois niveaux.

x Des sociétés marocaines ont ainsi pu accéder, en 1996, aux marchés internationaux, en émettant des actions par l'intermédiaire de certificats de titres mondiaux de dépôts, ou en émettant des obligations en Europe. Cf. Jbili et alii (1997).

xi En 1996, la capitalisation boursière représente $23 \%$ du PIB au Maroc et $21 \%$ en Tunisie. En quelques années le marché marocain dont le chiffre d'affaires a atteint 2,3 milliards de dollars en 1996 s'est hissé au deuxième rang de l'Afrique derrière l'Afrique du Sud et devant l'Egypte. En Tunisie, après une forte croissance 
au début des année 90, l'année 1996 a été marquée par une chute d'activité accompagnant une diminution de l'indice.

xii 18 sociétés ont été radiées de la cote en 1995, dans le cadre de l'opération d'assainissement décidée pour répondre aux exigences en matière de transparence et de liquidité.

xiii On n'en arrive pas encore à cette situation notée par Singh (1997) dans certains pays en développement où les sociétés recourent fortement au financement externe par actions, en contradiction avec le pecking order, du fait du coût relativement bas de l'appel aux actionnaires suite à l'envolée du cours des actions avec la libéralisation interne et l'afflux des investisseurs institutionnels étrangers.

xiv En Tunisie, par exemple, un rapport officiel sur la modernisation du système bancaire a souligné, en 1997 , les rigidités réglementaires qui subsistent, la fragilité des structures financières, particulièrement pour les banques publiques, et le fait qu'en dépit de marges importantes les établissements sont peu rentables. Cf.Krimi.

xv Ainsi la limite de participation étrangère fixée à $10 \%$ du capital des sociétés cotées à Tunis a-t-elle été portée à $49 \%$ et le rapatriement des dividendes et les mouvements de capitaux doivent y être totalement libérés à l'instar du Maroc.

xvi Cf. A. Alami, Le Monde, 8 avril 1997. 